\title{
The validity and morphology of lung mites of the genus Pneumocoptes (Acari: Astigmata)
}

\section{G.A. Kouchakji and B. Loos-Frank}

Z Parasitenkd (1984) 70:673-683

Due to an unfortunate error, part of the legend of Fig. 14 was omitted. It should have read as follows:

Fig. 14a, b. Micrographs demonstrating the difference in the digitus fixus dentition in a $P$. penrosei and $\mathbf{b} P$. jellisoni. Note the difference in the $\mathrm{Y}$-shaped apodemes of coxae $\mathrm{I}$ 